\title{
and Minimization of Total Error Rate in Cooperative Spectrum Sensing for Cognitive Radio Networks
}

\author{
Samit Kumar Ghosh ${ }^{1}$, Sachin Ravikant Trankatwar ${ }^{1}$, and P. Bachan ${ }^{2}$ \\ ${ }^{1}$ Department of EEE, BITS, Pilani, Hyderabad Campus, Hyderabad, India \\ ${ }^{2}$ Department of ECE, GLA University, Mathura, India
}

https://doi.org/10.26636/jtit.2021.144420

\begin{abstract}
In cognitive radio technology, spectrum sensing is essential for detecting spectrum holes which may be allotted to secondary users. In this paper, an optimal voting rule is used for cooperative spectrum sensing while minimizing the total error rate (TER). The proposed spectrum sensing method is more energy-efficient and may be implemented in practice. It is relied upon in an improved energy detector whose utilization depends on the presence or absence of the primary user. Expressions for false alarm and missed detection probabilities are derived in the paper as well. Overall performance is analyzed both for AWGN and Rayleigh fading channels, in the presence of additive white Gaussian noise (AWGN). The optimum voting rule is applied to the cooperative spectrum sensing process in order to identify the optimum number of sensing nodes and the detection threshold. Finally, an energy-efficient spectrum sensing algorithm is proposed, requiring a lower number of cognitive users for a given error bound.
\end{abstract}

Keywords-cognitive radio, energy detection, optimization, spectrum sensing.

\section{Introduction}

Efficient utilization of the spectrum is a primary requirement in current and future wireless communication systems. Exponential increase in the number of mobile services and smartphones requires a continued expansion of the spectrum resources [1], [2]. Hence, wireless multicasting techniques are used to transmit the same data to a group of users connected to the same base station, as such an approach is spectrally more efficient compared to wireless unicasting techniques [3]. Spectral efficiency may also be increased by using the non-utilized licensed spectrum assigned to primary users (PU). These spectra are non-continuous and are allocated dynamically to secondary users (SU) [4], [5]. Cognitive radio (CR) has been considered as a promising technique with this respect. CR is a software-defined technology, where the unused spectrum or the spectrum holes of the radio band owned by PU are detected and then as- signed to SU for the transmission of their data [6], [7]. Spectrum sharing (SS) is another popular method. In this approach, SU is allowed to coexist with PU within the same band, without violating interference temperature (IT) restrictions [8], [9]. This technique is commonly known as the underlay spectrum sharing mode [10]. An optimized power allocation approach enhances SS capacity, both for PUs and cognitive radio network (CRN) [11]. This power allocation strategy is constrained by primary link outage probability (OP), which significantly improves the throughput of SU by considering the PU's QoS-related constraint, and hardly considering the IT constraint [12].

Spectrum hole sensing is one of the key problems of CR, as a single CR cannot sense the PU's spectrum holes reliably due to low SNR, multi-path fading, shadowing, and sensing time-related constraints. Hence, spectrum sensing needs to be performed by multiple CR users [13]-[15]. The cooperative spectrum sensing (CSS) mechanism is one of the most advanced spectrum sensing methods used in CR, where multiple SUs are allowed to use spectrum holes of the PU by relying on the co-operative mechanism [16]-[18]. In CSS, SUs sense the presence of the PU's spectrum holes and send the relevant information to the fusion center (FC), where the final decision is made. The primary objective of the CR is to utilize the unused spectrum of the PU without interfering with the PU, so that spectrum utilization may be improved [19]. In CR, energy detection is one of the most effective spectrum sensing methods, as it may compare the energy-related statistics of the received signal with a predefined threshold and is capable of deciding whether the spectrum is available or not [20]. Performance of the energy detection mechanism may be measured by relying on detection probability and false alarm probability [21], [22]. The analysis of this energy detector comes in handy for emerging applications, including ultra-wideband and cognitive radio technologies. Spectrum access may be increased in CR by reducing false alarm probability, and interference suffered by PU will de- 
crease as detection probability improves. However, the important task is to establish a relationship between the two probabilities i.e. the probability of detecting $\mathrm{P}_{d}$ and the probability of false alarm $\mathrm{P}_{f}$, by selecting an appropriate detection threshold. To ensure the best performance detection results, CSS is used. Multiple cognitive users (CU) are used and their observations are combined at the fusion center, using a voting rule.

The key contributions of this paper are as follows.

1. Analytical frameworks are formed for the optimality of CSS, utilizing the detecting channel in CR and the primary transmitter when energy detection and distributed decision fusion are applied to a CRN.

2. Optimal detection threshold is derived to minimize the total error rate (TER).

3. The optimum voting rule i.e. the optimal value of $n$ for the $n$-out-of-N is studied, both in AWGN and in the Rayleigh fading channel.

4. The optimal number of CRs are is required in CSS with large CRN to achieve the target error bound.

Following this introduction, the remaining part of the paper is organized as follows: Section 2 introduces the model of the system. Section 3 presents optimization of cooperative spectrum sensing, along with numerical results and discussions. Finally, conclusions are drawn in Section 4.

\section{System Model}

We consider a centralized CSS architecture in a CR network with an erroneous sensing/reporting channel, presented in Fig. 1, consisting of a PU, N number of CR (SUs), and an FC. All SUs coordinate and collaborate with each other and finally forward their local binary decisions (i.e. 0 or 1) to the FC through the erroneous reporting channel. Based on the received observations, the FC decides about spectrum access. Each CU performs spectrum sensing and, based on the presence or lack of presence of a PU, a binary deci-

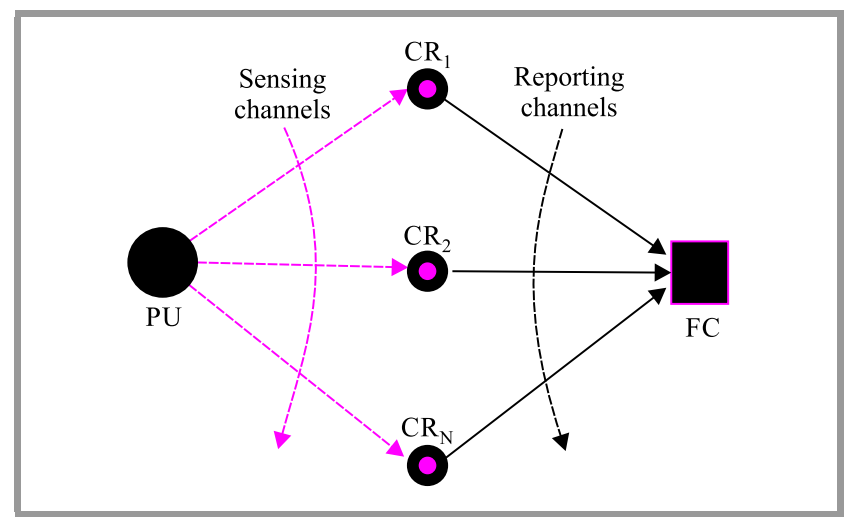

Fig. 1. Centralized CSS architecture in a CRN with erroneous reporting channel. sion is made between the subsequent two hypotheses $\left(H_{0}-\right.$ absence of PU, $H_{1}$ - presence of PU):

$$
r_{j}(t)=\left\{\begin{array}{ll}
n_{j}(t), & H_{0} \\
h_{j}(t) s(t)+n_{j}(t), & H_{1}
\end{array} .\right.
$$

In Eq. (1), $j$ is the antenna index, $j \in\{1,2, \ldots, \mathrm{N}\}$ at each $\mathrm{CR}, s(t)$ denotes the signal transmitted by the PU with energy $E_{s}, n_{j}(t) \sim \mathscr{C} \mathscr{N}\left(0, \sigma_{n}^{2}\right)$ is the circularly symmetric complex additive white Gaussian noise, where $\mathscr{C} \mathscr{N}($.$) denotes the complex normal distribution and$ $h_{j}(t) \sim \mathscr{C} \mathscr{N}\left(0, \sigma_{h}^{2}\right)$ represents the complex valued channel coefficient. Taking into account the assumption that, during the spectrum sensing process, sensing channel $h_{j}(t)$ is time-invariant and the activities of the PU remain unchanged, energy $r_{j}$ is taken as a decision statistic that has the following distribution:

$$
r_{j}= \begin{cases}\chi_{2 u}^{2}, & H_{0} \\ \chi_{2 u}^{2}\left(2 \gamma_{j}\right), & H_{1}\end{cases}
$$

where $\chi_{2 u}^{2}$ denotes a central chi-square distribution with $2 u$ degrees of freedom, which equals to twice the timebandwidth product $2 T W$, while $\chi_{2 u}^{2}\left(2 \gamma_{j}\right)$ denotes a noncentral chi-square distribution with the same number of degrees of freedom and a non-centrality parameter $2 \gamma_{j}$, which equals to twice the instantaneous SNR of the $j$-th CR. The average probability of false alarm $\mathrm{P}_{f, j}$, probability of detection $\mathrm{P}_{d, j}$, and probability of missed detection $\mathrm{P}_{m, j}$ over AWGN channels in the energy detector for $j$-th CR are given, respectively, by [23]:

$$
\begin{gathered}
\mathrm{P}_{f, j}=\frac{\Gamma\left(u, \frac{\lambda_{j}}{2}\right)}{\Gamma(u)}, \\
\mathrm{P}_{d, j}=Q_{u}\left(\sqrt{2 \gamma_{j}}, \sqrt{\lambda_{j}}\right)
\end{gathered}
$$

and

$$
\mathrm{P}_{m, j}=1-P_{d, j} .
$$

In Eqs. (3)-(4) parameters $u, \gamma_{i}$, and $\lambda_{i}$ signify the timebandwidth product, instantaneous signal-to-noise (SNR) ratio and energy detection threshold at the $j$-th CR, respectively. $\Gamma(a, b)$, and $Q_{u}(p, q)$ are the incomplete gamma function and the generalized Marcum Q-function, respectively [24], [25],

$$
\Gamma(a, b)=\int_{b}^{\infty} t^{a-1} \mathrm{e}^{-t} \mathrm{~d} t
$$

and

$$
Q_{u}(p, q)=\frac{1}{p^{u-1}} \int_{q}^{\infty} t^{u} \mathrm{e}^{-\frac{t^{2}+p^{2}}{2}} I_{u-1}(p t) \mathrm{d} t, \text { with } I_{u-1}(.)
$$

is the modified Bessel's function of $(u-1)$-th order. In wireless communication systems, $\mathrm{P}_{f}$ will depend on the channel only. Path distribution and SNR will not affect it. 
Hence, in Rayleigh fading distribution, $\mathrm{P}_{f}$ remains the same as AWGN in Eq. (3) and $\mathrm{P}_{d}$ is given by:

$$
\begin{aligned}
& \mathrm{P}_{d}=\mathrm{e}^{-\frac{\lambda}{2}} \sum_{n=0}^{u-1} \frac{1}{n !}\left(\frac{\lambda}{2}\right)^{n}+\left(\frac{1+\bar{\gamma}}{\bar{\gamma}}\right)^{u-1} \times \\
& {\left[\mathrm{e}^{-\frac{\lambda}{2(1+\bar{\gamma})}}-\mathrm{e}^{-\frac{-\lambda}{2}} \sum_{n=0}^{u-1} \frac{1}{n !}\left(\frac{\lambda \bar{\gamma}}{2(1+\bar{\gamma})}\right)^{n}\right] }
\end{aligned}
$$

Using the relation $\Gamma(a, x)=(a-1) ! \mathrm{e}^{-x} \sum_{n=0}^{a-1} \frac{x^{n}}{n !}$, i.e. a gamma function that is incomplete in terms of generalized Laguerre polynomials, the Eq. (6), may be written as:

$$
\begin{aligned}
\mathrm{P}_{d}=\left[\frac{\Gamma\left(u-1, \frac{\lambda}{2}\right)}{(u-2) !}\right]+ & \left(\frac{1+\bar{\gamma}}{\bar{\gamma}}\right)^{u-1} \mathrm{e}^{-\frac{\lambda}{2(1+\bar{\gamma})} \times} \\
& {\left[1-\frac{\Gamma\left(u-1, \frac{\lambda \bar{\gamma}}{2(1+\bar{\gamma})}\right)}{(u-2) !}\right] . }
\end{aligned}
$$

In CSS, the FC employs various hard decision-combining operations (OR, AND, majority rule) based on the received binary decision $D_{j} \in\{0,1\}$ ( 0 - absence of PU, 1 - presence of PU) and gives the final status (inactive/active) through the erroneous reporting channel. According to the $n$-out-of-N rule, all 1-bit decisions are fused together at the common receiver.

$$
\triangle=\sum_{j=1}^{\mathrm{N}} D_{j} \begin{cases}\geq n, & H_{1} \\ <n, & H_{0}\end{cases}
$$

where the integer $n$ is the threshold of the $n$-out-of-N voting rule that indicates the OR rule when $n=1$ and corresponds to the AND rule when $n=\mathrm{N}$. We assume that the distance between any two CRs is small compared to the distance from any $\mathrm{CR}$ to the primary transmitter, which implies that all CRs exhibit identical path losses. Therefore, we can conclude that in an AWGN environment, $\gamma_{1}=\gamma_{2}=\ldots \gamma_{\mathrm{N}}=$ $\gamma=\bar{\gamma}$ and in the case of Rayleigh fading, the instantaneous SNRs are identically distributed with their mean value of $\bar{\gamma}$. In fact, we suppose that all CRs use the same threshold $\lambda$, implying that $\lambda_{1}=\lambda_{2}=\ldots \lambda_{k}=\lambda$. This implies that $\mathrm{P}_{d, i}, \mathrm{P}_{f, i}$, and $\mathrm{P}_{m, i}$ being independent of $i$ in both fading and non-fading channels. Therefore, $\mathrm{P}_{d, i}=\mathrm{P}_{d}, \mathrm{P}_{f, i}=\mathrm{P}_{f}$, and $\mathrm{P}_{m, i}=\mathrm{P}_{m}$. For both kinds of environments, we have $\mathrm{P}_{m}=1-\mathrm{P}_{d}$. The false alarm and miss detection probability of CSS are expressed as:

$$
\begin{array}{r}
\mathrm{Q}_{f}=\mathrm{P}\left(H_{1} \mid H_{0}\right)=\sum_{l=n}^{\mathrm{N}}\left(\begin{array}{c}
\mathrm{N} \\
l
\end{array}\right) \mathrm{P}_{f}^{l}\left(1-\mathrm{P}_{f}\right)^{\mathrm{N}-l}, \\
\mathrm{Q}_{m}=\mathrm{P}\left(H_{0} \mid H_{1}\right)=1-\sum_{l=n}^{N}\left(\begin{array}{c}
\mathrm{N} \\
l
\end{array}\right) \mathrm{P}_{d}^{l}\left(1-\mathrm{P}_{d}\right)^{\mathrm{N}-l} .
\end{array}
$$

\section{Optimization of Cooperative Spectrum Sensing}

In this section, we study the optimality of CSS when energy detection and decision fusions are applied.

\subsection{Optimal Voting Rule}

An exact solution to obtain the optimal number of $\mathrm{CR}$ is required to minimize the TER i.e. $\mathrm{Q}_{f}+\mathrm{Q}_{m}$ based on the following theorem.

Theorem 1: Given fixed number of N, the optimal voting rule for cooperative spectrum sensing that minimizes $\mathrm{Q}_{f}+$ $\mathrm{Q}_{m}$ is $n_{\mathrm{opt}}=\min \left(\mathrm{N},\left\lceil\frac{\mathrm{N}}{1+\alpha}\right\rceil\right)$ where, $\alpha=\frac{\ln \frac{\mathrm{P}_{f}}{1-\mathrm{P}_{m}}}{\ln \frac{\mathrm{P}_{m}}{1-\mathrm{P}_{f}}}$ and $\lceil$. denotes the ceiling function.

Proof: Let $\mathrm{F}(n)$ be a function given by:

$$
\mathrm{F}(n)=\sum_{l=n}^{\mathrm{N}}\left(\begin{array}{c}
\mathrm{N} \\
l
\end{array}\right)\left[\mathrm{P}_{f}^{l}\left(1-\mathrm{P}_{f}\right)^{\mathrm{N}-l}-\left(1-\mathrm{P}_{m}\right)^{l} \mathrm{P}_{m}^{\mathrm{N}-l}\right] \text {. }
$$

Combining Eqs. (9) and (10), we get $\mathrm{Q}_{f}+\mathrm{Q}_{m}=1+\mathrm{F}(n)$. Then, we have

$$
\begin{aligned}
& \frac{\partial \mathrm{F}(n)}{\partial n} \approx \mathrm{F}(n+1)-\mathrm{F}(n) \\
&=\left(\begin{array}{l}
\mathrm{N} \\
n
\end{array}\right)\left[\left(1-\mathrm{P}_{m}\right)^{n} \mathrm{P}_{m}^{\mathrm{N}-n}-\mathrm{P}_{f}^{n}\left(1-\mathrm{P}_{f}\right)^{\mathrm{N}-n}\right] .
\end{aligned}
$$

The optimum value of $n$ is obtained when $\frac{\partial \mathrm{F}(n)}{\partial n}=0$, i.e. when

$$
\left(1-\mathrm{P}_{m}\right)^{n} \mathrm{P}_{m}^{\mathrm{N}-n}=\mathrm{P}_{f}^{n}\left(1-\mathrm{P}_{f}\right)^{\mathrm{N}-n}
$$

Let $\alpha=\frac{\ln \frac{\mathrm{P}_{f}}{1-\mathrm{P}_{m}}}{\ln \frac{\mathrm{P}_{m}}{1-\mathrm{P}_{f}}}$.

Then, after simplifying and re-arranging, we obtain $n \approx$ $\left\lceil\frac{N}{1+\alpha}\right\rceil$ which is a function of $\mathrm{P}_{f}$ and $\mathrm{P}_{m}$. From the proportion, we can conclude that:

1. The optimum value of $n$ is $\frac{\mathrm{N}}{2}$. This can be achieved when $\frac{\mathrm{P}_{f}}{\mathrm{P}_{m}}=1$, i.e. $\alpha=1$.

2. The OR rule is optimal when the parameter $\alpha \geq$ $\mathrm{N}-1$. This can be achieved when $\frac{\mathrm{P}_{f}}{\mathrm{P}_{m}} \ll 1$, i.e. for very large values of $\lambda$.

3. The AND rule is optimal when $\alpha=0$. This can be achieved when $\frac{\mathrm{P}_{f}}{\mathrm{P}_{m}} \gg 1$, i.e. for very small values of $\lambda$.

Here, we consider the 10 voting rules between $n=1$ and $n=10$ for $\mathrm{N}=10$ and calculate the TER at $\mathrm{SNR}=10 \mathrm{~dB}$ by considering the AWGN and Rayleigh fading channels. Figure 2 shows the plot of the TER versus the detection threshold, considering different voting rules between $n=1$ and $n=10$ in a CR network with 10 users, for both channels, respectively. It may be observed from Fig. $2 \mathrm{a}-\mathrm{b}$ that to achieve the minimum error optimum value of the threshold, $n=5$ for AWGN and $n=2$ for the Rayleigh fading channel. However, the OR rule i.e. $n=1$, and AND rule, i.e. $n=10$, tends to be optimal for very large and very small thresholds, respectively.

Table 1 presents the optimum values of $n$, threshold $(\lambda)$ and TER $\left(\mathrm{Q}_{f}+\mathrm{Q}_{m}\right)$, respectively, for various network parameters. From Table 1, it can be found that threshold 

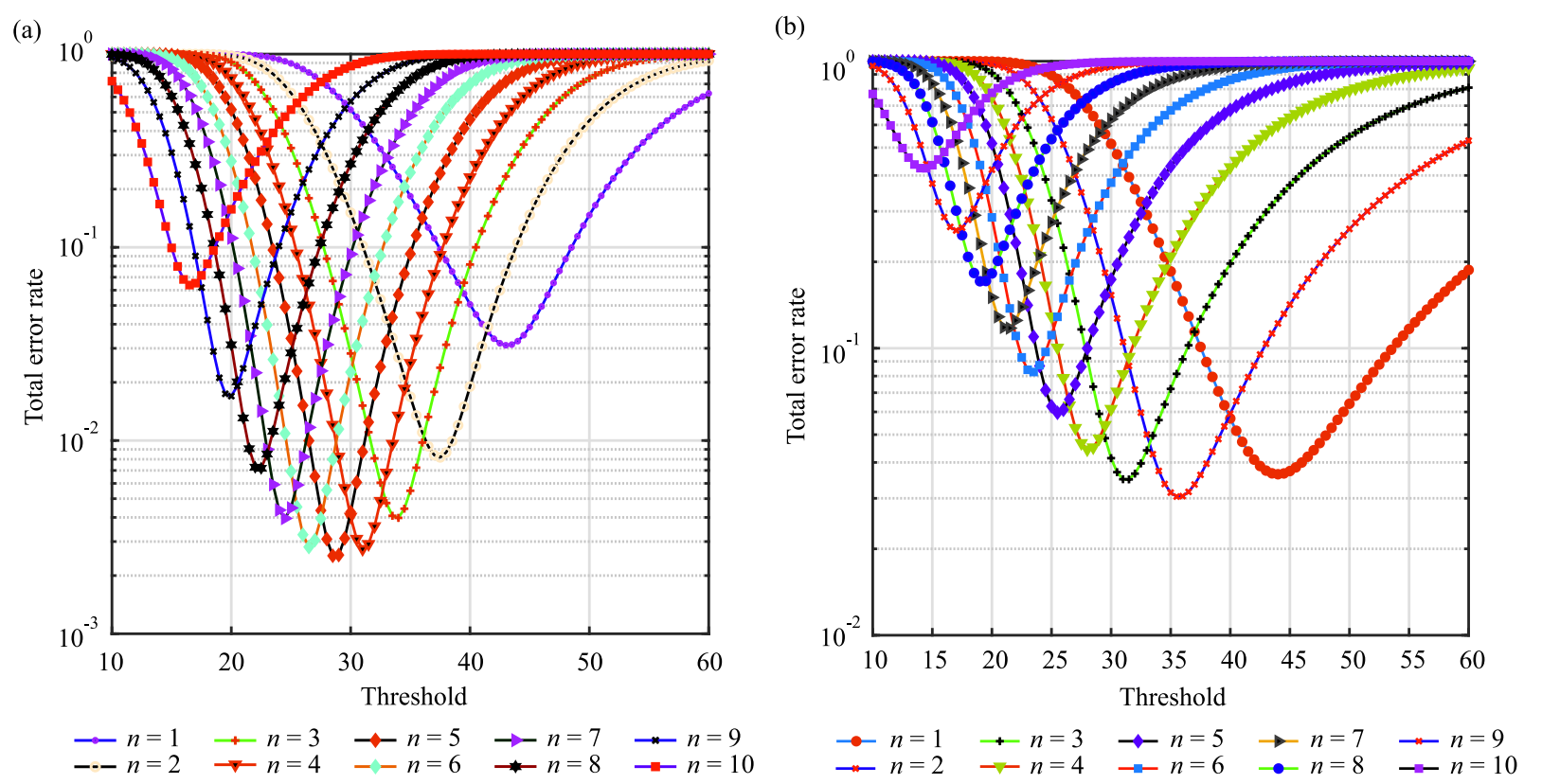

Fig. 2. Total error rate vs. threshold for AWGN channel (a) and total error rate vs. threshold for Rayleigh fading channel (b). (For color pictures see the digital version of the paper).

Table 1

Comparison of TER for different threshold values for $\mathrm{SNR}=10 \mathrm{~dB}$

\begin{tabular}{|c|c|c|c|c|c|c|c|c|c|c|c|}
\hline Channel & $n$ & 1 & 2 & 3 & 4 & 5 & 6 & 7 & 8 & 9 & 10 \\
\hline \hline \multirow{2}{*}{ AWGN } & Threshold & 43.0 & 37.5 & 34.0 & 31.0 & $\mathbf{2 8 . 5}$ & 26.5 & 24.5 & 22.5 & 20.0 & 17.0 \\
\cline { 2 - 12 } & TER & 0.0311 & 0.0082 & 0.0040 & 0.0027 & $\mathbf{0 . 0 0 2 5}$ & 0.0028 & 0.0040 & 0.0072 & 0.0170 & 0.0652 \\
\hline \multirow{2}{*}{ Rayleigh fading } & Threshold & 44.0 & $\mathbf{3 5 . 5}$ & 31.5 & 28.0 & 25.5 & 23.5 & 21.5 & 19.0 & 17.0 & 14.5 \\
\cline { 2 - 11 } & TER & 0.0364 & $\mathbf{0 . 0 3 0 4}$ & 0.0349 & 0.0444 & 0.0596 & 0.0826 & 0.1175 & 0.1711 & 0.2573 & 0.4236 \\
\hline
\end{tabular}

(a)

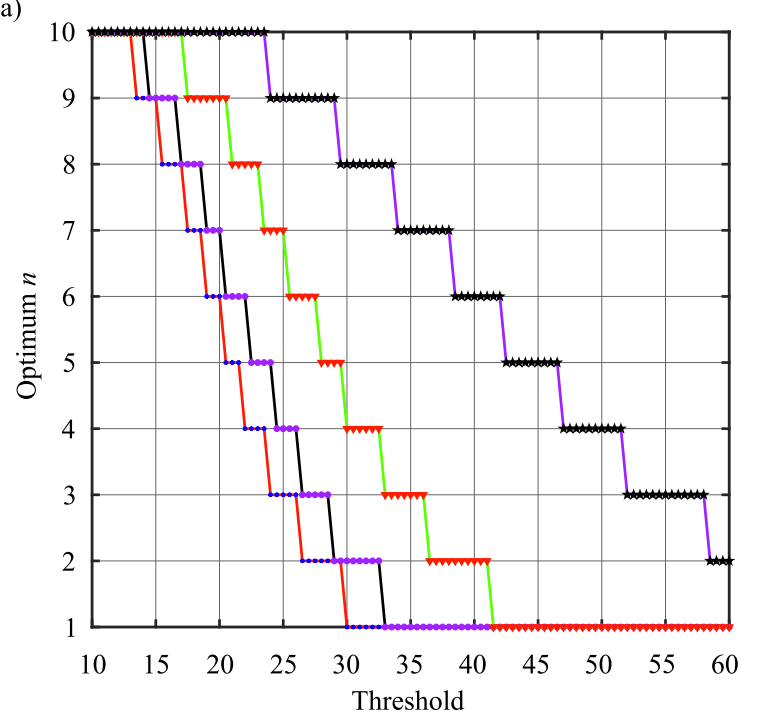

(b)

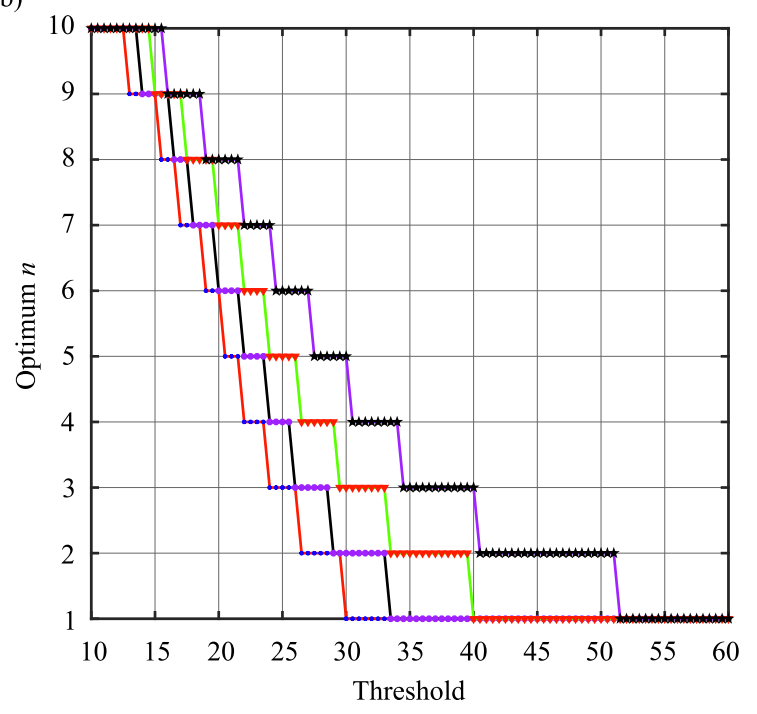

$\longrightarrow=0 \mathrm{~dB}$
$\longrightarrow \lambda=5 \mathrm{~dB}$
$\longrightarrow$

Fig. 3. Optimum voting rule vs. detection threshold for AWGN channel (a) and optimum voting rule vs. detection threshold for Rayleigh fading channel (b). 
values decrease as the number of cognitive users increases at $\mathrm{SNR}=10 \mathrm{~dB}$ in the case of both non-fading and fading channels. We also observe that minimization of TER occurs at $\lambda=28.5$ and $n=5$ in AWGN channel, and in the case of the Rayleigh fading channel, at $\lambda=35.5$ and $n=2$.

Figure $3 \mathrm{a}-\mathrm{b}$ shows the optimum voting rule versus detection threshold in the AWGN channel and the Rayleigh fading channel, respectively. It offers the exact solution of $n$ in terms of the detection threshold for $\mathrm{SNR}=0,5,10$, $15 \mathrm{~dB}$ and $\mathrm{N}=10$. It is evident that, as SNR increases, the optimum number of cooperative SUs increases, and that as the detection threshold increases, the optimum number of cooperative SUs decreases with the SNR.

\subsection{Optimum Energy Detection Threshold}

We could observe in Fig. 2a-b that the TER curve of $\mathrm{Q}_{f}+\mathrm{Q}_{m}$ in for a wide range of thresholds $\lambda$ for the AWGN channel and the Rayleigh fading channel, respectively, considering different voting rules from $n=1$ to 10 in a CR network. This indicates that for the minimization of $\mathrm{Q}_{f}+\mathrm{Q}_{m}$ only one value of $\lambda$ exists. The optimal threshold is given by $\lambda^{o p t}=\underset{\lambda}{\operatorname{minimized}}\left(\mathrm{Q}_{f}+\mathrm{Q}_{m}\right)$, which is achieved when $\frac{\partial \mathrm{Q}_{m}}{\partial \lambda}+\frac{\partial \mathrm{Q}_{f}}{\partial \lambda}=0$

From Eq. (9) we can obtain the following:

$$
\begin{aligned}
& \frac{\partial \mathrm{Q}_{f}}{\partial \lambda}=\sum_{l=n}^{\mathrm{N}}\left(\begin{array}{c}
\mathrm{N} \\
l
\end{array}\right) l \mathrm{P}_{f}^{l-1} \frac{\partial \mathrm{P}_{f}}{\partial \lambda}\left(1-\mathrm{P}_{f}\right)^{\mathrm{N}-l} \\
&-\sum_{l=n}^{\mathrm{N}}\left(\begin{array}{c}
\mathrm{N} \\
l
\end{array}\right) \mathrm{P}_{f}^{l}(\mathrm{~N}-l)\left(1-\mathrm{P}_{f}\right)^{\mathrm{N}-l-1} \frac{\partial \mathrm{P}_{f}}{\partial \lambda} \\
&=\frac{\partial \mathrm{P}_{f}}{\partial \lambda} \sum_{l=n}^{\mathrm{N}}\left(\begin{array}{c}
\mathrm{N} \\
l
\end{array}\right) \mathrm{P}_{f}^{l-1}\left(1-\mathrm{P}_{f}\right)^{\mathrm{N}-1} \\
& \times\left[l-(\mathrm{N}-l) \frac{\mathrm{P}_{f}}{1-\mathrm{P}_{f}}\right] .
\end{aligned}
$$

From Eq. (3), we obtain $\frac{\partial \mathrm{P}_{f}}{\partial \lambda}$ :

$$
\frac{\partial \mathrm{P}_{f}}{\partial \lambda}=\frac{\partial}{\partial \lambda} \frac{\Gamma\left(u, \frac{\lambda}{2}\right)}{\Gamma(u)}=-\frac{1}{(u-1) !} \frac{\lambda^{u-1}}{2^{u}} \mathrm{e}^{-\frac{\lambda}{2}} .
$$

From Eq. (10), we can get:

$$
\begin{aligned}
\frac{\partial \mathrm{Q}_{m}}{\partial \lambda}=- & \sum_{l=n}^{\mathrm{N}}\left(\begin{array}{c}
\mathrm{N} \\
l
\end{array}\right) l \mathrm{P}_{d}^{l-1} \frac{\partial \mathrm{P}_{d}}{\partial \lambda}\left(1-\mathrm{P}_{f}\right)^{\mathrm{N}-l} \\
& +\sum_{l=n}^{\mathrm{N}}\left(\begin{array}{c}
\mathrm{N} \\
l
\end{array}\right) \mathrm{P}_{d}^{l}(\mathrm{~N}-l)\left(1-\mathrm{P}_{d}\right)^{\mathrm{N}-l-1} \frac{\partial \mathrm{P}_{d}}{\partial \lambda} \\
& =-\frac{\partial \mathrm{P}_{d}}{\partial \lambda} \sum_{l=n}^{\mathrm{N}}\left(\begin{array}{c}
\mathrm{N} \\
l
\end{array}\right) \mathrm{P}_{d}^{l-1}\left(1-\mathrm{P}_{d}\right)^{\mathrm{N}-1} \\
& \times\left[l-(\mathrm{N}-l) \frac{\mathrm{P}_{d}}{1-\mathrm{P}_{d}}\right] .
\end{aligned}
$$

The detection probability of the AWGN channel is [23]:

$$
\begin{aligned}
\mathrm{P}_{d}=\mathrm{Q}_{u}(\sqrt{2 \gamma}, \sqrt{\lambda}) \\
=\frac{1}{(\sqrt{2 \gamma})^{u-1}} \int_{\sqrt{\lambda}}^{\infty} x^{u} \mathrm{e}^{-\frac{x^{2}+2 \gamma}{2}} I_{u-1} \sqrt{2 \gamma} x \mathrm{~d} x .
\end{aligned}
$$

From Eq. (17), we obtain:

$$
\frac{\partial \mathrm{P}_{d}}{\partial \lambda}=-\frac{\lambda^{\frac{u-1}{2}}}{2(2 \gamma)^{\frac{u-1}{2}}} \mathrm{e}^{-\frac{\lambda+2 \gamma}{2}} I_{u-1}(\sqrt{2 \gamma \lambda}) .
$$

The detection probability of the Rayleigh fading channel is given in Eq. (7). Note that, $\Gamma(a, x)=\int_{x}^{\infty} t^{a-1} \mathrm{e}^{-t} \mathrm{dt}$ and $\frac{\partial \Gamma(a, x)}{\partial x}=-x^{a-1} \mathrm{e}^{-x}$.

Then:

$$
\begin{gathered}
\frac{\partial \Gamma\left(u-1, \frac{\lambda}{2}\right)}{\partial \lambda}=-\frac{1}{2}\left(\frac{\lambda}{2}\right)^{u-2} \mathrm{e}^{-\frac{\lambda}{2}} \\
\frac{\partial \Gamma\left(u-1, \frac{\lambda \bar{\gamma}}{2(1+\bar{\gamma})}\right)}{\partial \lambda}=-\frac{1}{2} \frac{\bar{\gamma}}{1+\bar{\gamma}}\left(\frac{\lambda \bar{\gamma}}{2(1+\bar{\gamma})}\right)^{u-2} \mathrm{e}^{-\frac{\lambda \bar{\gamma}}{2(1+\bar{\gamma})}} .
\end{gathered}
$$

Now consider

$$
\mathrm{A}_{1}=\Gamma\left(u-1, \frac{\lambda}{2}\right)
$$

and

$$
\mathrm{B}_{1}=\Gamma\left(u-1, \frac{\lambda \bar{\gamma}}{2(1+\bar{\gamma})}\right)
$$

By differentiating Eq. (7) w.r.t $\lambda$, we get:

$$
\begin{aligned}
\frac{\partial \mathrm{P}_{d}}{\partial \lambda} & =\frac{\frac{\partial \mathrm{A}_{1}}{\partial \lambda}}{(u-2) !}+\left(\frac{1+\bar{\gamma}}{\bar{\gamma}}\right)^{u-1} \frac{\mathrm{e}^{-\frac{\lambda}{2(1+\bar{\gamma})}}}{2(1+\bar{\gamma})} \\
& {\left[1-\frac{\mathrm{B}_{1}}{(u-2) !}\right]-\frac{\frac{\partial \mathrm{B}_{1}}{\partial \lambda}}{(u-2) !}\left(\frac{1+\bar{\gamma}}{\bar{\gamma}}\right)^{u-1} \mathrm{e}^{-\frac{\lambda}{2(1+\bar{\gamma})}} . }
\end{aligned}
$$

Simplifying first term and the third term, using Eqs. (19) and (20) in Eq. (21), we can write:

$$
\frac{\partial \mathrm{P}_{d}}{\partial \lambda}=\left(\frac{1+\bar{\gamma}}{\bar{\gamma}}\right)^{u-1} \frac{\mathrm{e}^{-\frac{\lambda}{2(1+\bar{\gamma})}}}{2(1+\bar{\gamma})}\left[\frac{\Gamma\left(u-1, \frac{\lambda \bar{\gamma}}{2(1+\bar{\gamma})}\right)}{(u-2) !}-1\right]_{(22)}
$$

Further, using Eq. (7), we obtain:

$$
\frac{\partial \mathrm{P}_{d}}{\partial \lambda}=\frac{1}{2(1+\bar{\gamma})}\left[\frac{\Gamma\left(u-1, \frac{\lambda}{2}\right)}{(u-2) !}-\mathrm{P}_{d}\right]
$$

The solution to $\frac{\partial \mathrm{Q}_{m}}{\partial \lambda}+\frac{\partial \mathrm{Q}_{f}}{\partial \lambda}=0$ (for $\lambda$ ) can be calculated using Eqs. (14) and (16). 

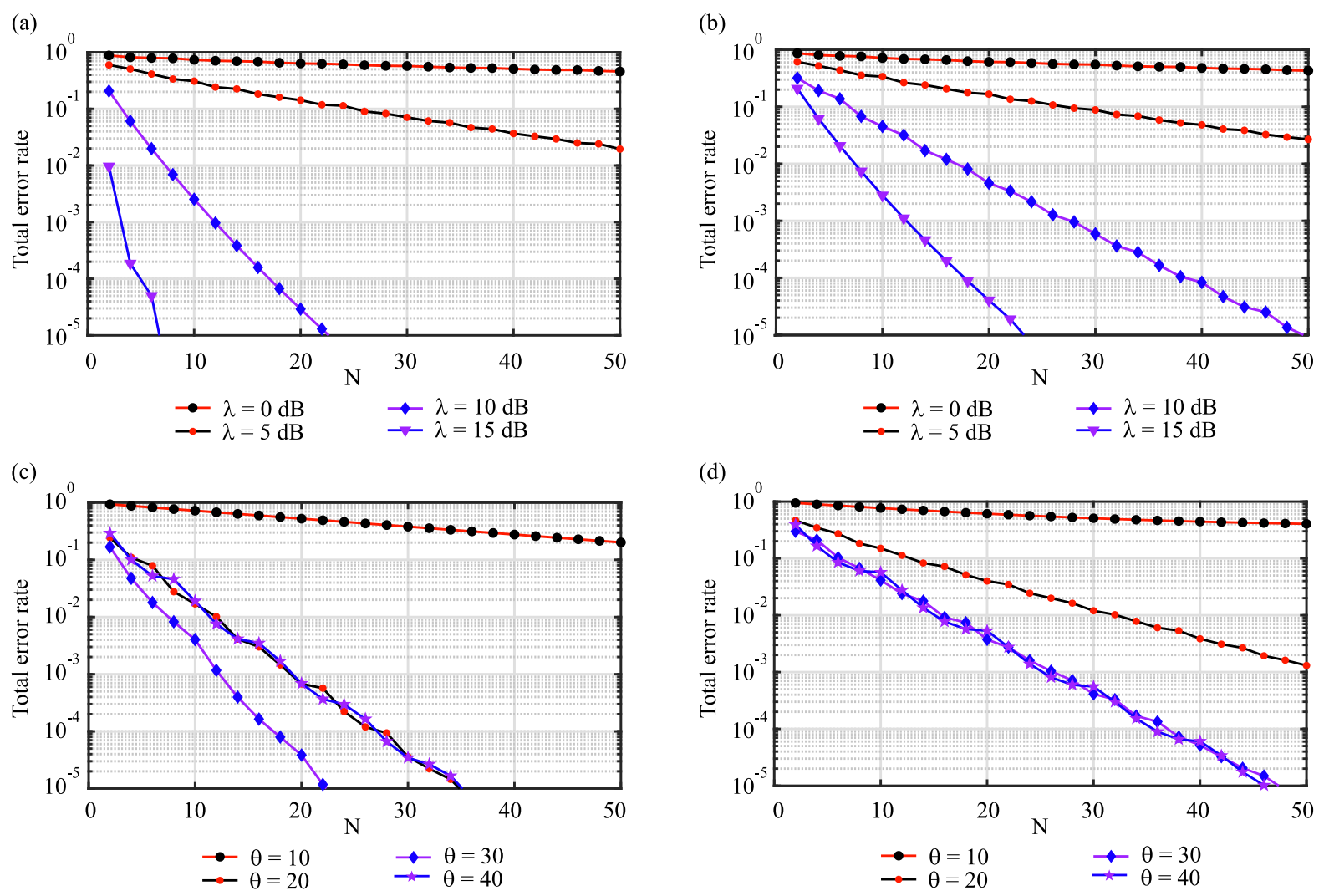

Fig. 4. Total error rate of CSS versus number of collaborating CRs in a network with $50 \mathrm{CRs}$ in: (a) AWGN with SNR $=0-20 \mathrm{~dB}$ for $\lambda=28.5$, (b) Rayleigh fading with SNR $=0-20 \mathrm{~dB}$ for $\lambda=28.5$, (c) AWGN with SNR $=10 \mathrm{~dB}$ for $\lambda=10-40$, (d) Rayleigh fading with SNR $=10 \mathrm{~dB}$ for $\lambda=10-40$.

\subsection{Optimal Number of Cognitive Radios}

CSS become impractical when the number of CRs is higher in a CRN. So, for a particular specified time period, only one CR is capable of sending its local decision to the common receiver and of splitting the decision without difficulty at the end of the receiver. This problem may be tended to by enabling the CRs to transmit their choices simultaneously. But this causes difficulties related to the design of the receiver at the time of sorting out the decisions from various types of CRs.

Another possible option is to make choices concerning orthogonal frequency ranges, but that involves a significant portion of the usable bandwidth.

To resolve these problems, we suggest an effective sensing algorithm that relies on the transmission of judgment for one CR using one time slot, but retains a minimum error bound by allowing a few CRs in CSS, instead of all of them. First of all, we assume that to satisfy $\mathrm{Q}_{m}+\mathrm{Q}_{f} \leq \varepsilon$, the minimum number of CRs required in CSS is $\tilde{n}^{*}\left(1 \leq \tilde{n}^{*} \leq \mathrm{N}\right)$. The optimal voting rule obtained from Theorem 1 for CSS with $\tilde{n}^{*} \mathrm{CR}$ is $n_{\tilde{n}^{*}}^{o p t}=\min \left(\tilde{n}^{*},\left[\frac{\tilde{n}^{*}}{1+\alpha}\right]\right)$ where $\alpha$ is related to $\mathrm{P}_{f}$ and $\mathrm{P}_{m}$, and may be evaluated by giving the value of $\lambda$ and the SNR. Let us define function $\mathrm{F}(.,$.$) in terms of$ variable as $\mathrm{F}\left(\tilde{n}, n_{\tilde{n}}^{o p t}\right)=\mathrm{Q}_{f}+\mathrm{Q}_{m}-\varepsilon$, where $\tilde{n}$ denotes the number of cooperative CRs in CSS. Probabilities $\mathrm{Q}_{f}$ and
$\mathrm{Q}_{m}$ are the functions of $\tilde{n}$, and and $n_{\tilde{n}}^{o p t}$ are given by Eqs. (9) and (10), respectively. Then, we have $\mathrm{F}\left(\tilde{n}, n_{\tilde{n}}^{\text {opt }}\right) \leq 0$ and $\mathrm{F}\left(\tilde{n}-1, n_{\tilde{n}-1}^{o p t}\right) \geq 0$.

Using these properties, we can obtain $\tilde{n}^{*}=\left\lceil\tilde{n}_{0}\right\rceil$, where $\tilde{n}_{0}$ represents the first zero-crossing point of the curve $\mathrm{F}\left(\tilde{n}, n_{\tilde{n}}^{o p t}\right)$ in terms of $\tilde{n}$. Therefore, it is possible to formulate a rapid spectrum sensing algorithm by only considering $\tilde{n}^{*}$ in CSS instead of N. As a result, the duration of sensing can be reduced from $\mathrm{N}$ time slots to $\tilde{n}^{*}$ time slots, while this error bound $\varepsilon$ is guaranteed. To achieve a TER $\mathrm{Q}_{f}+\mathrm{Q}_{m}<0.01$, the smallest number of CRs required is 2 and 7 for SNR values of $15 \mathrm{~dB}$ and $10 \mathrm{~dB}$, respectively, with a fixed decision threshold of $\lambda=28.5$ over the AWGN channel. However, in the Rayleigh fading channel, the minimum values of CRs are 7 for $15 \mathrm{~dB}$ and 17 for $10 \mathrm{~dB}$. Figure 4 shows the CSS error rate according to $\mathrm{Q}_{f}(\mathrm{~N}, n)+\mathrm{Q}_{m}(\mathrm{~N}, n)$ with respect to specific $N$ when the optimum voting rule $n=n_{\mathrm{N}}^{\text {opt }}=\min \left(\mathrm{N},\left\lceil\frac{\mathrm{N}}{1+\alpha}\right\rceil\right)$ is implemented. This implies that it is necessary to employ limited cooperation in order to achieve the level of service needed.

Figure $4 \mathrm{c}$ and Fig. $4 \mathrm{~d}$ demonstrate another example. It is observed that at SNR values of $10 \mathrm{~dB}$ and for the given error rate of 0.01 , the number of CRs considered for threshold values of 30,40 , and 20 is 7,11 , and 12, respectively for 
the AWGN channel, and 15, 16, and 32 for the threshold values of 40,30 , and 20 .

\section{Conclusion}

In this work, we investigated the performance of cooperative spectrum sensing with energy detection. We derive the expression for the optimum value of $n$ that minimizes the TER by applying the $n$-out-of-N voting rule. The numerical expression for obtained the optimal detection threshold obtained has been discussed as well. In addition, an effective spectrum sensing algorithm has been proposed that needs less than the total number of cognitive radios in the cooperative spectrum sensing, thus fulfilling the specified bound error.

\section{References}

[1] S. Haykin, "Cognitive radio: brain-empowered wireless communications", IEEE J. on Selec. Areas in Commun., vol. 23, no. 2, pp. 201-220, 2005 (DOI: 10.1109/JSAC.2004.839380).

[2] Y.-C. Liang, Y. Zeng, E. C. Peh, and A. T. Hoang, "Sensingthroughput tradeoff for cognitive radio networks", IEEE Trans. on Wirel. Commun., vol. 7, no. 4, pp. 1326-1337, 2008 (DOI: 10.1109/TWC.2008.060869).

[3] K. Poularakis, G. Iosifidis, V. Sourlas, and L. Tassiulas, "Exploiting caching and multicast for $5 \mathrm{G}$ wireless networks", IEEE Trans. on Wirel. Commun., vol. 15, no. 4, pp. 2995-3007, 2016 (DOI: 10.1109/TWC.2016.2514418).

[4] F. Haider et al., "Spectral and energy efficiency analysis for cognitive radio networks", IEEE Trans. on Wirel. Commun., vol. 14, no. 6 , pp. 2969-2980, 2015 (DOI: 10.1109/TWC.2015.2398864).

[5] M. Klymash, M. Jo, T. Maksymyuk, and I. Beliaiev, "Spectral efficiency increasing of cognitive radio networks", in Proc. 12th Int. Conf. on the Exper. of Desig. and Appl. of CAD Syst. in Microelectron. CADSM 2013, Polyana Svalyava, Ukraine 2013, pp. 169-171 (ISBN: 9781467364614).

[6] J. Mitola and G. Q. Maguire, "Cognitive radio: making software radios more personal”, IEEE Pers. Commun., vol. 6, no. 4, pp. 13-18, 1999 (DOI: 10.1109/98.788210).

[7] B. Wang and K. R. Liu, "Advances in cognitive radio networks: A survey", IEEE J. of Selec. Topics in Sig. Process., vol. 5, no. 1, pp. 5-23, 2010 (DOI: 10.1109/JSTSP.2010.2093210).

[8] T. Yucek and H. Arslan, "A survey of spectrum sensing algorithms for cognitive radio applications", IEEE Commun. Surv. \& Tutor., vol. 11 , no. 1 , pp. 116-130, 2009 (DOI: 10.1109/SURV.2009.090109).

[9] M. S. Falih and H. N. Abdullah, "A combined spectrum sensing method based DCT for cognitive radio system", Int. J. of Elec. \& Comp. Engin., vol. 10, no. 2, pp. 1935-1942, 2020 (DOI: 10.11591/ijece.v10i2.pp1935-1942).

[10] I. F. Akyildiz, W.-Y. Lee, M. C. Vuran, and S. Mohanty, "Next generation/dynamic spectrum access/cognitive radio wireless networks: A survey", Computer Networks, vol. 50, no. 13, pp. 2127-2159, 2006 (DOI: 10.1016/j.comnet.2006.05.001).

[11] X. Kang, Y.-C. Liang, A. Nallanathan, H. K. Garg, and R. Zhang, "Optimal power allocation for fading channels in cognitive radio networks: Ergodic capacity and outage capacity", IEEE Trans. on Wirel. Commun., vol. 8, no. 2, pp. 940-950, 2009 (DOI: 10.1109/TWC.2009.071448).

[12] K. B. Letaief and W. Zhang, "Cooperative communications for cognitive radio networks", Proc. of the IEEE, vol. 97, no. 5, pp. 878-893, 2009 (DOI: 10.1109/JPROC.2009.2015716).
[13] G. Zhao, G. Y. Li, C. Yang, and J. Ma, "Proactive detection of spectrum holes in cognitive radio", in Proc. of IEEE Int. Conf. on Commun., Dresden, Germany, 2009 (DOI: 10.1109/JPROC.2009.2015716).

[14] W. Zhang, R. K. Mallik, and K. B. Letaief, "Optimization of cooperative spectrum sensing with energy detection in cognitive radio networks", IEEE Trans. on Wirel. Commun., vol. 8, no. 12, pp. 5761-5766, 2009 (DOI: 10.1109/TWC.2009.12.081710).

[15] S. K. Ghosh and P. Bachan, "Performance evaluation of spectrum sensing techniques in cognitive radio network", IOSR J. of Electron. and Commun. Engin. (IOSR-JECE), vol. 12, no. 4, pp. 17-21, 2017 (DOI: 10.9790/2834-1204051721).

[16] I. F. Akyildiz, B. F. Lo, and R. Balakrishnan, "Cooperative spectrum sensing in cognitive radio networks: A survey", Phys. Commun., vol. 4, no. 1, pp. 40-62, 2011 (DOI: 10.1016/j.phycom.2010.12.003).

[17] G. Verma and O. Sahu, "A distance based reliable cooperative spectrum sensing algorithm in cognitive radio", Wirel. Pers. Commun., vol. 99, no. 1 , pp. 203-212, 2018 (DOI: $10.1007 / \mathrm{s} 11277-017-5052-\mathrm{z})$.

[18] P. Bachan, S. K. Ghosh, and S. K. Saraswat, "Comparative error rate analysis of cooperative spectrum sensing in non-fading and fading environments", in Proc. of Commun., Contr. and Intell. Syst. CCIS 2015, Mathura, India, 2015 pp. 124-127, 2015 (DOI: 10.1109/CCIntelS.2015.7437891).

[19] W. Zhang and K. B. Letaief, "Cooperative spectrum sensing with transmit and relay diversity in cognitive radio networks", IEEE Trans. on Wirel. Commun., vol. 7, no. 12, pp. 4761-4766, 2008 (DOI: 10.1109/T-WC.2008.060857).

[20] S. K. Ghosh, J. Mehedi, and U. C. Samal, "Sensing performance of energy detector in cognitive radio networks", Int. J. of Inf. Technol., vol. 11 , no. 4 , pp. 773-778, 2019 (DOI: $10.1007 / \mathrm{s} 41870-018-0236-7)$.

[21] S. Atapattu, C. Tellambura, and H. Jiang, "Energy detection based cooperative spectrum sensing in cognitive radio networks", IEEE Trans. on Wirel. Commun., vol. 10, no. 4, pp. 1232-1241, 2011 (DOI: 10.1109/TWC.2011.012411.100611).

[22] G. Mahendru, A. Shukla, and P. Banerjee, "A novel mathematical model for energy detection based spectrum sensing in cognitive radio networks", Wirel. Pers. Commun., vol. 110, no. 3, pp. 1237-1249, 2020 (DOI: 10.1007/s11277-019-06783-3).

[23] F. F. Digham, M.-S. Alouini, and M. K. Simon, "On the energy detection of unknown signals over fading channels", IEEE Trans. on Commun., vo. 55, no. 1, pp. 21-24, 2007 (DOI: 10.1109/TCOMM.2006.887483).

[24] I. S. Gradshteyn and I. M. Ryzhik, Table of Integrals, Series, and Products, 8th ed. Academic Press, 2014 (ISBN: 9780123849335).

[25] A. Nuttall, "Some integrals involving the Q_M function (Corresp.)", IEEE Trans. on Inform. Theory, vol. 21, no. 1, pp. 95-96, 1975 (DOI: 10.1109/TIT.1975.1055327).

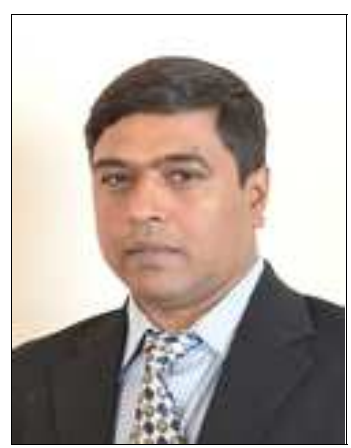

Samit Kumar Ghosh received his B.Tech. degree in Electronics and Communication Engineering from Kalyani University, West Bengal, India, in 2002 and the M.Tech. degree in Electronics System and Communication Engineering from the National Institute of Technology (NIT) Rourkela, Rourkela, Odisha, India, in 2006. Currently, he is pursuing the Ph.D. degree in the department of Electrical and Electronics Engineering (EEE), Birla Institute of Technology and Science, Pilani, Hyderabad Campus, Hyderabad, India. His research interests include biomedical signal processing, medical devices and 
instrumentation, non-stationary signal processing, machine learning, cognitive radio, spectrum sensing. He has published research papers in reputed international journals and conferences.

(iD) https://orcid.org/0000-0003-2267-7314

E-mail:samitnitrkl@gmail.com

Department of Electrical and Electronics

Engineering

Birla Institute of Technology and Science, Pilani

Hyderabad Campus, Hyderabad-500078

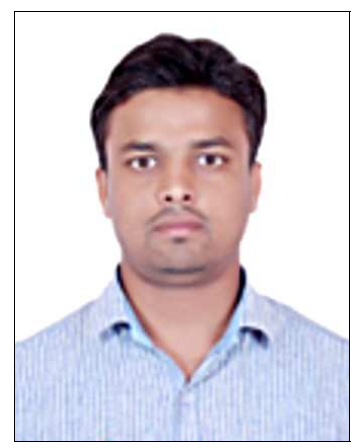

Sachin Ravikant Trankatwar received his B.E. degree in Electronics and Telecommunications Engineering from IETE, New Delhi, in 2010, and a Master's degree from Electronics and Telecommunications Engineering Department, DBATU, Lonere, Raigad, India, in 2013. $\mathrm{He}$ is currently pursuing a Ph.D. degree from BITS Pilani, Hyderabad Campus, Hyderabad, India. He has over five years of teaching experience at an undergraduate level. His current research interest includes efficient techniques for $5 \mathrm{G}$ cellular networks. https://orcid.org/0000-0001-5114-2058

E-mail:strankatwar@gmail.com

Department of Electrical and Electronics Engineering

Birla Institute of Technology and Science, Pilani

Hyderabad Campus, Hyderabad-500078

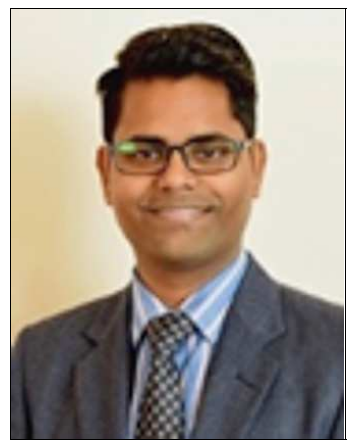

P. Bachan received his B.Tech. from Dayalbagh Educational Institute, Agra, India in 2007, M.Tech. from National Institute of Technology, Kurukshetra, India in 2010. He is currently working as an Assistant Professor and also pursuing his Ph.D. in the Department of Electronics and Communication Engineering, GLA University, Mathura, India. His research interest includes spectrum sensing, multiple access techniques for next gen networks, channel estimation techniques and massive MIMO.

(iD) https://orcid.org/0000-0001-8188-5901

E-mail:p.bachan@gmail.com

Department of Electronics and Communication

Engineering

GLA University, Mathura

Mathura-281 406 (U.P.) 